\title{
Precision Measurement of Total Column Carbon Dioxide using a Space Borne Fabry-Perot Interferometer
}

\author{
William S. Heaps \\ Laser \& Electro-optics Branch, NASA Goddard Space flight Center. Greenbelt, MD 20771 \\ Phone: 301-286-5106 FAX: 301-286-1750 E-mail: wheaps@pop500.gsfc.nasa.gov
}

\begin{abstract}
A design is proposed for a space borne Fabry-Perot interferometer targeted explicitly for the measurement of the total column of $\mathrm{CO}_{2}$ having a precision better than 1 part in 370 . (c) 2000 Optical Society of America

OCIS codes: (010.1280)Atmospheric Composition (120.2230) Fabry-Perot
\end{abstract}

\section{Introduction}

Understanding the Global Carbon Cycle has become a high priority within NASA and the earth science community. It is now widely understood that atmospheric $\mathrm{CO}_{2}$ is a greenhouse gas absorbing outgoing IR radiation from the earth and re-radiating this energy isotropically with a net reduction in outward energy flux. It is also recognized that the total amount of $\mathrm{CO}_{2}$ in the atmosphere has been increasing steadily for decades and likely will continue to do so since it is an end product of combustion which scales with global population. There have been a number of international meetings held recently to discuss what global action should be undertaken to ameliorate this huge potential for global climate modification. Because the direct restriction of $\mathrm{CO}_{2}$ emissions has very harsh economic penalties in both the developed and undeveloped countries it is very desirable that mankind acquire an improved understanding of the budget for $\mathrm{CO}_{2}$ so that alternative strategies might be employed. A significant step in achieving this understanding would be a series of global maps of $\mathrm{CO}_{2}$ delimiting the spatial and temporal variations of this gas and allowing the identification of source regions and sink regions around the globe. At the present time the global average abundance of $\mathrm{CO}_{2}$ is on the order of 360-370 parts per million (ppm). It is estimated that a $\mathrm{CO}_{2}$ source or sink at the surface might generate a fluctuation in the background $\mathrm{CO}_{2}$ abundance on the order of $10-20 \mathrm{ppm}$. However the effect of this change when viewed from space through the entire column of $\mathrm{CO}_{2}$ would produce a column average change of only about $1 \mathrm{ppm}$. This means in order for an instrument viewing from space to detect a source or sink region it must have a precision of at least 1 in 370 .

\section{Some spectroscopy}

The basis for the instrument proposed herein is spectral analysis of solar flux reflected off the surface of the earth. The total column for carbon dioxide is about $6.4 \times 10^{21}$ molecules per $\mathrm{cm}^{2}$. For best sensitivity to changes in this column one would like the absorption for two passes through the atmosphere to be on the order of $10 \%$ or so. This means the desired absorption cross section should be on the order of 10-23. Carbon dioxide has a number of absorption bands in the IR from 1.4 microns out to beyond 10 microns. Two bands, the 30013-00001 centered at $6228 \mathrm{~cm}-1$ and the 30012-00001 centered at $6345 \mathrm{~cm}-1$ have lines with absorption cross sections in this range. They are also in regions of the spectrum which are relatively free from lines arising from other species especially water vapor. We have selected the 30012-00001 band for this design. A small portion of this band is reproduced in Fig. 1 .

\section{Fabry-Perot Interferometers}

Fabry-Perot interferometer consists of two flat, parallel, partially reflecting plates. Interference occurs in the gap between the plates such that only wavelengths which fit an exact integral number of times in the gap between the two plates are transmitted. Because this condition can be satisfied for many different integers simultaneously the passband for a Fabry-Perot interferometer consists of a series of narrow lines separated by a constant energy difference extending as far spectrally as the transmission of the plates and the reflectivity of the plate coatings permit. The constant energy separation between these passbands is termed the free spectral range (FSR). The spectral width of a single transmission peak depends upon the reflectivity of the plates. High reflectivity corresponds to a narrow spectral bandpass. The ratio of the FSR to the full width half maximum (FWHM) of s single passband is termed the finesse of the Fabry-Perot. High finesse means a narrow spectral passband. 


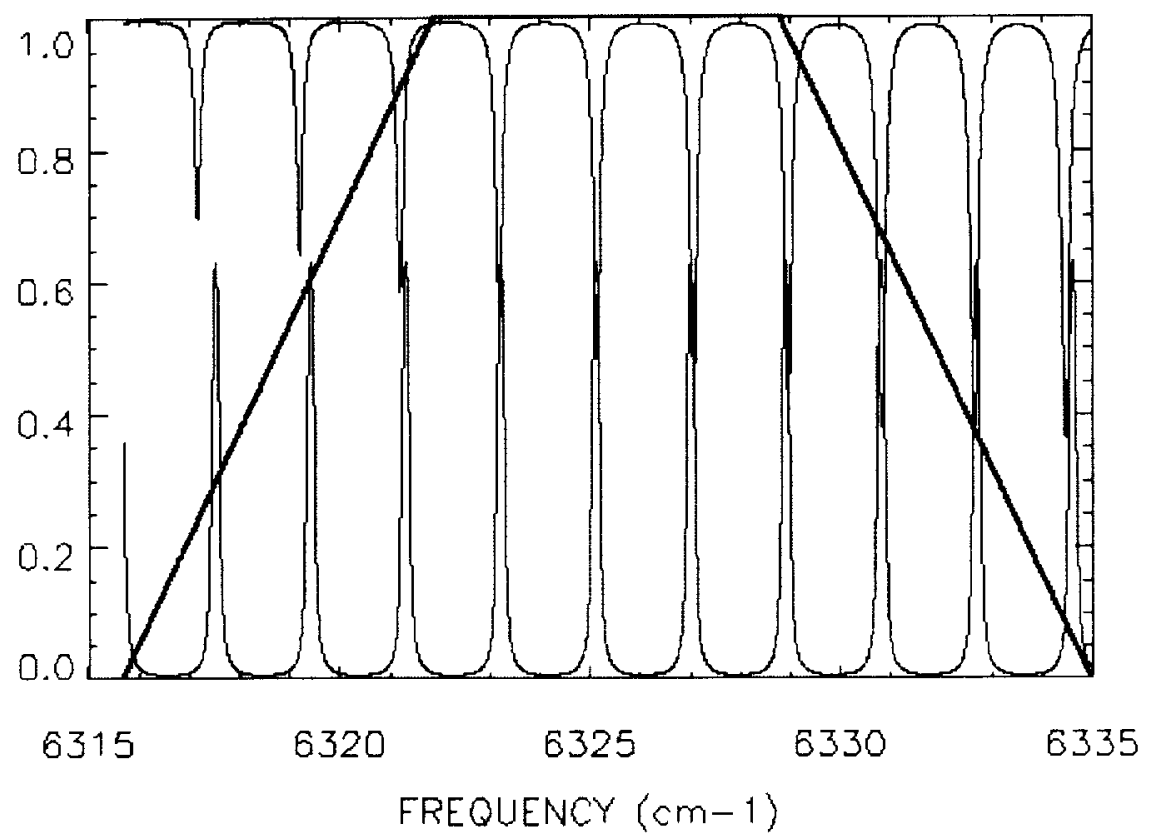

Fig. 1. A portion of the $\mathrm{P}$ branch of the 30012-00001 band of $\mathrm{CO}_{2}$ is shown descending from the top. The corresponding passbands of the FabryPerot ascend from below. The prefilter has a trapezoidal shape.

The passband for a Fabry-Perot can be tuned by changing the optical length between the Fabry-Perot plates. This has been effected in a number of ways. The plates themselves can be physically moved using some type of precision actuators such as piezo-electric stacks. The index of refraction of the gap can be changed by changing pressure or the composition of the gas between the plates. If the Fabry-Perot is implemented by coating the opposite sides of a single transparent plate then tuning can be accomplished by changing the index of the plate material with temperature.

For most applications of Fabry-Perots the multiple passbands have been something of an inconvenience. Indeed in order to isolate a single wavelength for investigation Fabry-Perots have been used together with grating spectrometers or several Fabry-Perots have been operated in tandem. In this application however we use the fact that the multiple passbands of the Fabry-Perot have an equal energy spacing and that the multiple $\mathrm{CO}_{2}$ lines in the absorption band are almost equally spaced. The first benefit that this offers is that more light can get through the instrument. In order to achieve the very high precision we require the signal to noise ratio (SNR) of the instrument must be very high. More light means a higher SNR.

The second benefit that this approach offers is to reduce the sensitivity of the instrument to temperature changes. The various lines shown in the figure above arise from different energy levels of the $\mathrm{CO}_{2}$ molecule which have a relative population that depends on temperature. Lines near the right hand side of the figure arise from low lying energy levels which lose population as the molecule warms up while those on the left side arise from energy levels that gain population with temperature. This is illustrated in Fig. 2.

By careful selection of the prefilter passband several lines of each type can be included in the instrument's regard such that the overall effect of changing temperature has a much smaller effect on the effective absorption of the instrument. The temperature response of the instrument diagrammed in Fig. 1. is shown in Fig. 3. 


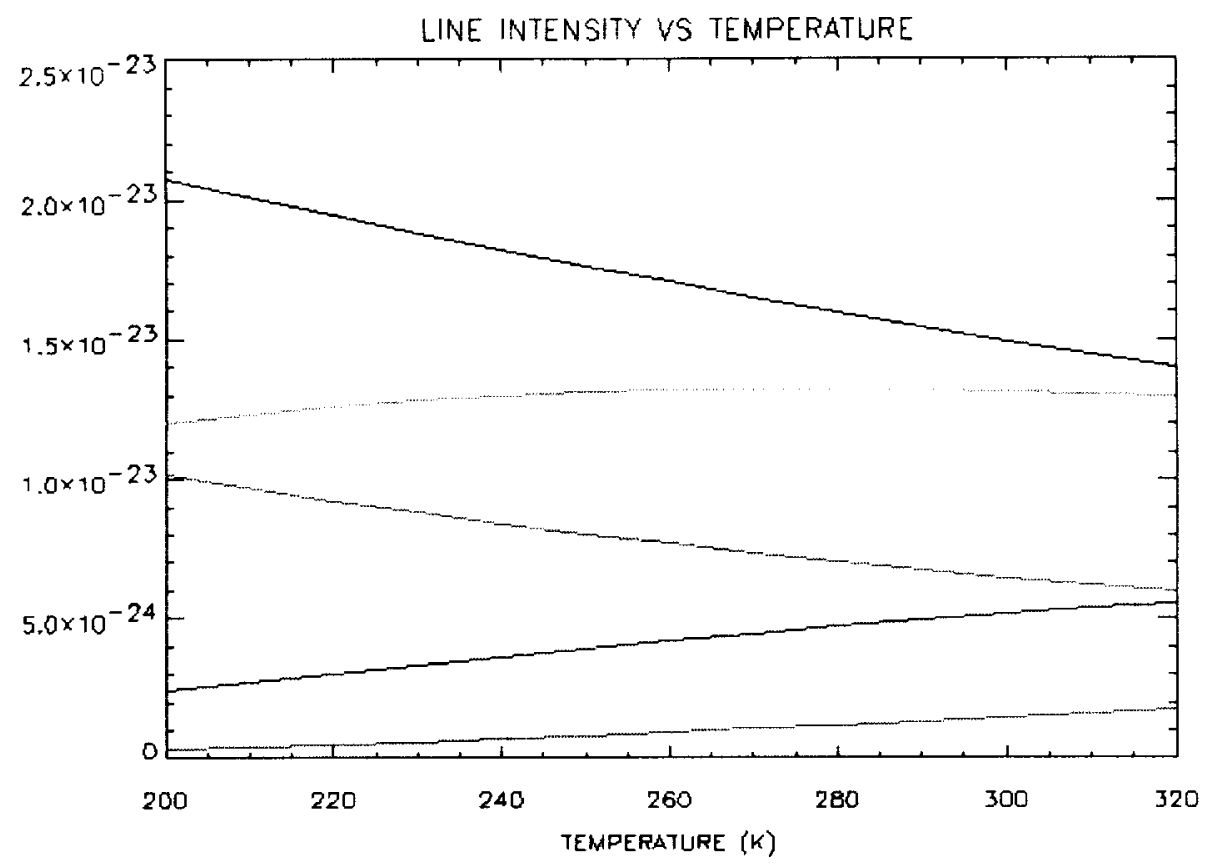

Fig. 2. The temperature dependence of several lines from the $\mathrm{P}$ branch is illustrated. Lines arising from low lying energy levels get weaker.

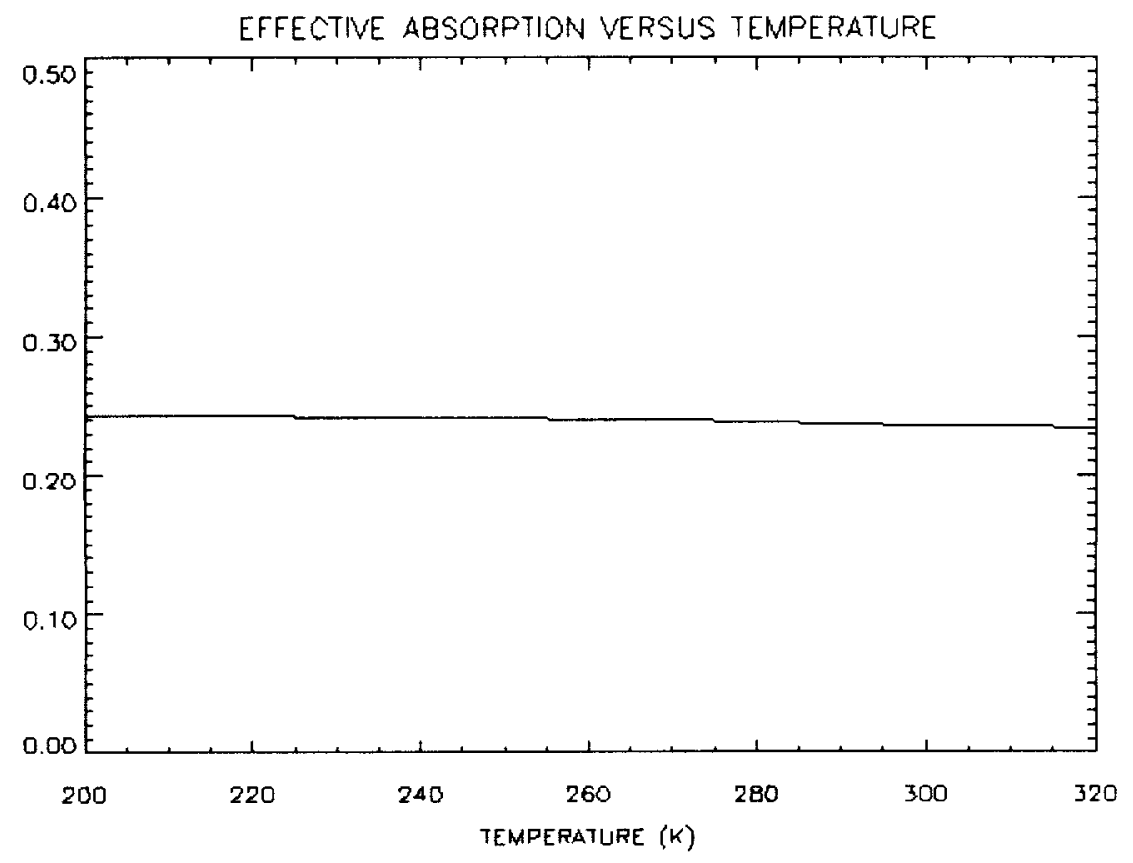

Fig. 3. The effective change in absorption with temperature for a group of $\mathrm{CO}_{2}$ lines viewed through the Fabry-Perot

\section{Conclusion}

It is shown that using a Fabry-Perot interferometer to analyze multiple atmospheric absorption lines simultaneously can improve the performance of a space borne absorption instrument over previously implemented devices. Additional considerations such as Doppler effects due to earth and satellite motion as well as interference by water vapor and other species will also be discussed. It is concluded that an instrument based upon the Fabry-Perot interferometer could be implemented and would offer the requisite precision. 